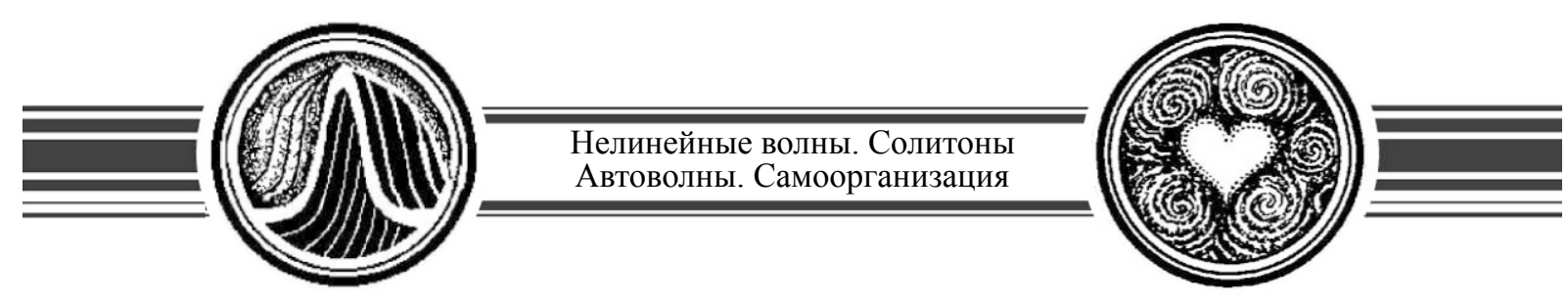

УДК 541.12

https://doi.org/10.18500/0869-6632-2019-27-6-73-90

\title{
Нелинейные температурные волны: Анализ на основе нелинейного уравнения теплопроводности
}

\author{
М. В. Давидович ${ }^{1}$, И. А. Корнев ${ }^{2}$, А. И. Тимофеев ${ }^{2}$ \\ ${ }^{1}$ Саратовский национальный исследовательский государственный университет имени Н.Г. Чернышевского \\ Россия, 410012 Саратов, Астраханская, 83 \\ ${ }^{2}$ ООО НПП «Этна Плюс» \\ Россия, 410040 Саратов, Вишнёвая, 11 \\ E-mail: davidovichMV@info.sgu.ru, iak.etna@yandex.ru, anton_timofeev@list.ru \\ Автор для переписки Давидович Михаил Владимирович, davidovichMV@info.sgu.ru \\ Поступила в редакцию 17.03.2019, принята к публикаичи 19.10.2019, опубликована 2.12.2019
}

Цель работы - ввести в рассмотрение нелинейное уравнение теплопроводности, в котором учтено излучение по закону Стефана-Больцмана внутри структуры с каждой виртуальной поверхности (что предполагает введение вариации коэффициента черноты тела), и на его основе рассмотреть температурные волны. Исследуемые модели. Изучается нелинейная волна в плоском одномерном хорошо прозрачном слое в декартовой системе координат с большим градиентом температуры и термостатами на границах. Считается, что вариация коэффициента черноты не зависит от координаты и температуры. Рассмотрена также модель остывания цилиндрического объема с водой в теплоизолирующей оболочке. Результаты. Получено нелинейное уравнение теплопроводности, основанное на балансе энергии, которое применено к прозрачной для излучения области с градиентом температуры. Проведено численное исследование движения температурных волн, демонстрирующее сильные нелинейные свойства: увеличение крутизны фронта без возможности опрокидывания, рост скорости волны при увеличении градиента температуры. Показано, что учет излучения важен для динамики остывания даже при невысоких температурах и в рассмотренной задаче приводит к увеличению расчетной скорости остывания на несколько десятков процентов. Приведены и обсуждены границы применимости уравнения и моделей. Обсуждение. В методическом плане предлагаемый материал может быть интересен для инженеров, студентов и аспирантов, занимающихся теплофизикой. Результаты могут быть применены для расчета тепловых процессов в прозрачных атмосферах небесных тел, а также для анализа температурных полей в микро- и наноструктурах, например, при разогреве автоэмиссионных структур.

Ключевые слова: уравнение лучистой теплопроводности, закон Стефана-Больцмана, закон Кирхгофа, коэффициент черноты, нелинейные температурные волны.

Образец ицитирования: Давидович М.В., Корнев И.А., Тимофеев А.И. Нелинейные температурные волны: Анализ на основе нелинейного уравнения теплопроводности // Известия вузов. ПНД. 2019. Т. 27, № 6. С. 73-90. https://doi.org/10.18500/0869-6632-2019-27-6-73-90

Финансовая поддержка. Работа выполнена при поддержке РНФ, проект № 16-19-10033. 


\title{
Nonlinear temperature waves: Analysis based on the nonlinear heat equation
}

\author{
M. V. Davidovich ${ }^{1}$, I. A. Kornev ${ }^{2}$, A. B. Timofeev ${ }^{2}$ \\ ${ }^{1}$ Saratov State University \\ 83 Astrakhanskaya, Saratov 410012, Russia \\ ${ }^{2}$ Research and Production Company «Etna Plus» \\ 11 Vishnevaya, Saratov 410040, Russua \\ E-mail: davidovichMV@info.sgu.ru, iak.etna@yandex.ru, anton timofeev@list.ru \\ Correspondence should be addressed to Michael V. Davidovich, davidovichMV@info.sgu.ru \\ Received 17.03.2019, accepted for publication 19.10.2019, published 2.12.2019
}

\begin{abstract}
The aim of the work is to introduce a nonlinear equation of thermal conductivity, which takes into account the radiation according to the Stefan-Boltzmann law inside the structure from each virtual surface (which assumes the introduction of body-blackness coefficient variation), and on its basis to consider temperature waves. Studied model. A nonlinear wave in a plane one-dimensional well-transparent layer in a Cartesian coordinate system with a large temperature gradient and thermostats at the boundaries is studied. It is believed that the variation of the blackness coefficient does not depend on the coordinate and temperature. The model of cooling of a cylindrical volume with water in a heat-insulating shell is also considered. Results. Nonlinear equation of thermal conductivity based on the energy balance is obtained, which is applied to a region transparent to radiation with a temperature gradient. Numerical study of temperature waves, showing a strong nonlinear properties: steepness increase of the front without possibility of overturning, the increase in wave velocity with increasing temperature gradient. It is also shown that accounting for radiation is important for cooling dynamics even at low temperatures, and in the considered problem leads to an increase in the calculated cooling rate by several tens of percent. Discussion. Limits of applicability of the equation and models are given and discussed. In terms of methodology, the proposed material may be of interest to engineers, students and postgraduates engaged in thermophysics. Results can be applied to calculation of thermal processes in transparent atmospheres of celestial bodies, as well as to analysis of temperature fields in micro-and nanostructures, for example, during heating of auto-emission structures.
\end{abstract}

Key words: equation of radiant heat transfer, Stefan-Boltzmann law, Kirchhoff law, blackness coefficient, nonlinear temperature waves.

Reference: Davidovich M.V., Kornev I.A., Timofeev A.I. Nonlinear temperature waves: Analysis based on the nonlinear heat equation. Izvestiya VUZ. Applied Nonlinear Dynamics, 2019, vol. 27, no. 6, pp. 73-90.

https://doi.org/10.18500/0869-6632-2019-27-6-73-90

Acknowledgements. The work was supported by Russian Science Foundation, project no. 16-19-10033.

\section{Введение}

Обычно в литературе рассматривают уравнения теплопроводности, считая, что теплопередача осуществляется непосредственным взаимодействием атомов, с обменом тепловыми фононами. Это требует введения коэффициента теплопроводности $k$, плотности вещества $\rho$ и коэффициента теплоемкости $c_{0}$. Если указанные величины не зависят от температуры $T$, линейное уравнение теплопроводности (ЛУТ) является дифференциальным уравнением (ДУ) параболического типа [1-6]. В случае введения зависимости от температуры уравнение теплопроводности становится нелинейным [1-6]. Обычно перенос тепла за счет теплопроводности и радиационный или лучистый перенос тепла рассматриваются раздельно на основе уравнений разных типов [7-11], а именно имеют место уравнения переноса лучистой энергии [12-20]. Лучистый (радиационный) перенос характерен для сильно нагретых тел (например, плазмы звезд): хотя теплопроводность их плазмы весьма высока, перенос за счет излучения может существенно преобладать. Обычно плотность лучистой энергии при этом весьма высока и должна быть учтена. Лучистый перенос также характерен для нагретой и достаточно разреженной (то есть достаточно прозрачной) плазмы, где взаимодействие частиц за счет столкновений не столь существенно, 
а плотность тепловой энергии вещества может быть существенно меньше равновесной плотности лучистой энергии $E_{p}=4 \sigma T^{4} / c$. Характерный пример - солнечная корона. Перенос излучения рассматривается как совокупность актов испускания, движения и поглощения фотонов атомами, поэтому он идет со скоростью света в веществе. Здесь мы не рассматриваем явления конвекции. В монографии [20] выведено и рассмотрено уравнение лучистой теплопроводности (УЛТ) $\partial Q / \partial t=F-\nabla \cdot S$ в форме закона сохранения энергии с введением коэффициента лучистой теплопроводности $k=16 l_{R} \sigma T^{3} / 3$. В этом уравнении $Q$ - полная плотность тепловой энергии; $S=-l_{R} c \nabla E_{p} / 3$ - поверхностная плотность его потока, связанная с равновесной плотностью энергии теплового излучения $E_{p} ; F$ - плотность тепловых источников; $T$ - абсолютная температура; $\sigma=5.67 \cdot 10^{-8} \mathrm{BT} /\left(\mathrm{M}^{-2} \cdot \mathrm{K}^{-4}\right)$ - постоянная Стефана-Больцмана; $l_{R}$ - росселандов пробег излучения. Усреднение по Росселанду - усреднение по температурной производной планковской спектральной плотности излучения. Пробег зависит от поглощающей способности вещества, спектрального состава излучения и скорости его переноса. Обычно последнюю считают не зависящей от вещества и равной скорости света в вакууме $c$, что справедливо для квантов очень больших энергий. Уравнение лучистой теплопроводности является приближенным, оно получено в диффузионном приближении в предположении квазиравновесности и имеет нелинейность четвертого порядка.

Удобно рассмотреть уравнения, единым образом описывающие теплопроводность и излучение. Такие нелинейные уравнения теплопроводности (НУТ), основанные только на балансе тепла (на уравнении непрерывности для его потока), были введены в работах $[21,22]$ для задач цилиндрических и сферических конфигураций. В них введен поток излучения $S=\sigma \varepsilon T^{4}$ в телесный угол $2 \pi$, а сами уравнения также имеют четвертый порядок по температуре. Нелинейные уравнения теплопроводности основаны на законе сохранении тепловой энергии и на том, что плотность потока тепла в секунду $q$ через единичную площадку в направлении нормали $v$, обычно выражаемая по закону Фурье как $q=-k v \cdot \nabla u$, заменяется на $q=-k v \cdot \nabla u+\delta \varepsilon \sigma u^{4}$, то есть к потоку за счет теплопроводности добавляется радиационный поток. Здесь и далее величины $u(r, t) \equiv T(r, t)$ означают температуру, $k(r)$ - коэффициент теплопроводности, $\delta \varepsilon(\mathbf{r})=$ $=\varepsilon(\mathbf{r}-0)-\varepsilon(\mathbf{r}+0)=\varepsilon^{+}(\mathbf{r})-\varepsilon^{-}(\mathbf{r})-$ вариация коэффициента черноты (ВКЧ), заключенного в пределах $0<\varepsilon(\mathbf{r})<1$, которая также может зависеть от температуры. Сам коэффициент черноты понимается как усредненный по планковскому спектру. Для уравнения лучистой теплопроводности $\mathbf{q}=-k(\mathbf{r}) \nabla u(\mathbf{r})-(l c / 3) \nabla E_{p}(\mathbf{r})$, где $E_{p}(\mathbf{r})=4 \sigma u^{4}(\mathbf{r}) / c-$ плотность энергии излучения, то есть уравнение имеет четвертый порядок нелинейности. Если градиента температуры нет, то в общем случае $q \neq 0$, поскольку поток $\delta \varepsilon \sigma u^{4}=\left(\varepsilon^{+}-\varepsilon^{-}\right) \sigma u^{4}$ двусторонний и идет, согласно закону Кирхгофа, от более черного тела к менее черному, то есть возникает разность потоков, $\nabla \cdot\left(v \delta \varepsilon \sigma u^{4}\right)$, в бесконечно тонком слое. В связи с этим имеют место нелинейные члены с $u^{4}$ и $u^{3} \nabla u$. Для нелинейного уравнения теплопроводности вместо длины пробега по Росселанду следует использовать длину пробега $l$, усредненную по планковской спектральной функции. Тогда, для выполнения условия квазиравновесности и применимости приведенных ниже соотношений должно выполняться условие $\left|\partial_{x} u(x)\right| \ll|u(x+l)-u(x)| / l$ для каждой координаты $x$.

\section{1. Постановка задачи и основные соотношения}

Выполняя баланс энергии для плоского слоя вещества $(x, x+\Delta x)$ и переходя к пределу $\Delta x \rightarrow 0$, получаем одномерное нелинейное уравнение теплопроводности в декартовых координатах

$$
w(x) \partial_{t} u(x, t)=\left(k(x) u^{\prime}(x, t)\right)^{\prime}-\sigma \Phi\left(u(x, t), u^{\prime}(x, t), \delta \varepsilon(x), \delta \varepsilon^{\prime}(x)\right)+F(x, t) .
$$

Здесь $w(x)=E / u=c_{0}(x) \rho(x), \rho(x)$ - плотность, $c_{0}(x)$ - теплоемкость, $F(x, t)$ - плотность 
мощности источников тепла, $E$ - плотность тепловой энергия вещества, штрихом обозначена производная по координате, а нелинейная оператор-функция имеет вид

$$
\Phi(u(x), \varepsilon(x))=4 \delta \varepsilon(x) u^{3}(x, t) u^{\prime}(x, t)+u^{4}(x, t) \varepsilon^{\prime}(x) .
$$

Здесь пренебрегаем плотностью энергии излучения, что оправдано для не слишком сильно нагретых тел. В общем случае $E=c_{0} \rho u+4 \sigma u^{4} / c[20,23]$. Считается, что ВКЧ $\delta \varepsilon$ не зависит от температуры. Зависимость от температуры добавит к $\Phi$ член $u^{4}(\partial \delta \varepsilon / \partial u)(\partial u / \partial x)$, что не принципиально. Нелинейный член возникает за счет дополнительного радиационного потока в слой $\left.\sigma \delta \varepsilon(x, u(x, t)) u^{4}(x, t)-\sigma \delta \varepsilon(x+\Delta x, u(x+\Delta x, t)) u^{4}(x+\Delta x, t)\right)$. При $\Delta x \rightarrow 0$ в нем достаточно удержать только члены с первыми производными в разложениях Тейлора. Входящие в (1) коэффициенты $k, \rho$ и $c_{0}$ также могут зависеть (и обычно зависят) от температуры, и тогда (1) есть НУТ даже в пренебрежении радиационным переносом. Обычно именно такие уравнения и называют НУТ $[1,6]$. В качестве $c_{0}$ понимают теплоемкость при постоянном давлении $c_{p}$. В трехмерном случае криволинейных координат следует рассмотреть баланс в слое, ограниченном двумя координатными (конгруэнтными) поверхностями $\Delta S(0)$ и $\Delta S(\delta)$, точки которых связаны по нормали $v(\mathbf{r}, t)=\nabla u(\mathbf{r}, t) /|\nabla u(\mathbf{r}, t)|$ расстоянием $\delta$ при $\delta \rightarrow 0, \Delta S \rightarrow 0$ (температура изменяется только в направлении нормали). Введем параметр с размерностью длины $\rho_{0}(\mathbf{r})=\Delta S /(\partial(\Delta S) / \partial \delta)$, характеризующий изменение площадей при смещении на $\delta$. В этом случае имеем

$$
c_{0}(\mathbf{r}) \rho(\mathbf{r}) \partial u(\mathbf{r}, t) / \partial t=\nabla \cdot(k(\mathbf{r}) \nabla u(\mathbf{r}, t))-\sigma \Phi(u(\mathbf{r}, t), \delta \varepsilon(\mathbf{r}, u(\mathbf{r}, t)))+F(\mathbf{r}, t),
$$

где оператор-функция принимает вид

$$
\begin{aligned}
& \Phi(u(\mathbf{r}, t), \varepsilon(\mathbf{r}, u(\mathbf{r}, t)))= \\
& =u^{4}(\mathbf{r}, t) \varepsilon(\mathbf{r}, u(r, t)) / \rho_{0}(r)+\left\{u^{4}(r, t) \nabla \delta \varepsilon(\mathbf{r}, u(\mathbf{r}, t))+\left[4 \varepsilon(\mathbf{r}, u(\mathbf{r}, t)) u^{3}(\mathbf{r}, t)+\right.\right. \\
& \left.\left.+u^{4}(\mathbf{r}, t) \partial \varepsilon(\mathbf{r}, u(\mathbf{r}, t)) / \partial u\right] \nabla u(r, t)\right\} \cdot v(r, t) .
\end{aligned}
$$

Очевидно, $\Phi=-\sigma \nabla \cdot\left(v(\mathbf{r}) \delta \varepsilon(\mathbf{r}) u^{4}(\mathbf{r})\right)$. Значения этой оператор-функции, полученные на решении ДУ (2), удобно далее обозначать как функцию координат и времени $\Phi(\mathbf{r}, t)$. В цилиндрической системе координат $r, \varphi, z$ при азимутально независимых решениях имеем $\rho_{0}=r$, а в сферической системе координат $r, \theta, \varphi$ при независимости решений от угла $\rho_{0}=r / 2$, поскольку $v=\mathbf{r} / r$. В общем случае $v=-\nabla u(\mathbf{r}) /|\nabla u(\mathbf{r})|$, и НУТ трехмерное. При делении на $a^{2}(\mathbf{r})=c_{0}(\mathbf{r}) \rho(\mathbf{r})$ уравнение (2) имеет вид нелинейного уравнения Шредингера (НУШ) с нелинейностью четвертого порядка, если теплоемкость, плотность и коэффициент черноты не зависят от температуры. В случае температурной зависимости всех коэффициентов нелинейность носит сложный характер. В случае изменения температуры только вдоль координат $x$ или $r$ имеем $|\nabla u(\mathbf{r}, t)|=\partial u / \partial x$ или $|\nabla u(\mathbf{r}, t)|=\partial u / \partial r$.

Отличие введенных уравнений от УЛТ следующее. В простейшем случае одномерного уравнения в декартовой системе нелинейный член в УЛТ $16 l \sigma u^{2}(x)\left(u^{\prime 2}(x)+u(x) u^{\prime \prime}(x) / 3\right)$, а в предложенном НУТ $-4 \sigma \delta \varepsilon u^{3}(x) u^{\prime}(x)$ (при условии, что $l$ и $\varepsilon$ постоянные). Знак нелинейного члена в первом случае определяется второй производной и он отрицателен только если она отрицательна и удовлетворяет условию $u^{\prime \prime}(x)<-3 u^{\prime 2}(x) / u(x)$. Знак нелинейного члена для НУТ противоположен знаку первой производной. Это означает, что поток тепла идет в сторону уменьшения температуры. Для УЛТ поток тепла определяется как $(-16 / 3) l \sigma u^{3}(x) u^{\prime}(x)$, а для НУТ как $\sigma \delta \varepsilon u^{4}(x)$. В обоих случаях излучение должно быть квазиравновесным. Здесь и далее под квазиравновесностью системы понимается, что скорость релаксации в области существенно больше, 
чем скорость изменения условий на ее границах. Под такой скоростью можно, например, понимать отношение втекающей в систему мощности к полной ее энергии. Уменьшая объем области до минимального, где это еще справедливо, получаем область, где справедливо понятие температуры. Для высокотемпературных процессов скорость релаксации увеличивается с температурой. Использование НУТ предполагает плавное изменение температуры и оценку степени черноты и ее вариации $\delta \varepsilon$, а для ЛУТ имеет место характерная длина $l$, которую нужно определять или как-то оценивать.

Рассмотрим вопрос, когда учет нелинейности в НУТ и в УЛТ существенен. При контакте черного тела с прозрачной средой в ней $\varepsilon^{+} \approx 1, \varepsilon^{-} \approx 0, \delta \varepsilon \sim 1$. В НУТ должно быть $k u^{\prime \prime} \sim$ $\sim-4 \sigma u^{3} u^{\prime}$. В этом случае обозначаем $T_{\sigma}=\sqrt[3]{k /\left(4 \sigma l_{0}\right)}$, где $l_{0}=\left|u^{\prime} / u^{\prime \prime}\right|$. Если $k=k_{0}(r) u^{\alpha}(r)$, то $T_{\sigma}=\sqrt[3-\alpha]{k /\left(4 \sigma l_{0}\right)}$. Функция $u^{\prime \prime}$ определяет кривизну распределения температуры, $u^{\prime}(x)-$ крутизну этого распределения, величина $l_{0}$ имеет размерность длины и связана со скоростью температурных волн, возникающих только за счет теплопроводности $V_{k}=(\partial u / \partial t) /(\partial u / \partial x)=$ $=a^{2} / l_{0}$. В УЛТ должно быть $16 l \sigma u^{2}(x)\left(u^{\prime 2}(x)+u(x) u^{\prime \prime}(x) / 3\right) \sim k u^{\prime \prime}(x)$. Пусть $u^{\prime}(x)=u(x) / l_{1}(x)$, где $l_{1}(x)$ - характерная длина изменения температуры. Тогда $u^{\prime 2}(x)+u(x) u^{\prime \prime}(x) / 3=(1 / 3+$ $\left.+l_{0}(x) / l_{1}(x)\right) u(x) u^{\prime \prime}(x)$ и $16 l \sigma u^{4}(x)\left(l_{0}(x) / l_{1}(x)+1 / 3\right) \sim k$. Обозначая $\mu=\left(1 / 3+l_{0}(x) / l_{1}(x)\right)$, имеем характерную температуру $T_{c}=\sqrt[4]{k /(l \sigma \mu) / 2}$.

Рассмотрим скорость движения температуры. ЛУТ не является релятивистски инвариантным, поэтому температура от возникающего в момент $t_{0}$ источника распространяется с бесконечной скоростью, что определяет функция Грина $(Ф Г) ~[1,6]$. При $t>t_{0}$ возбуждение существует везде, но сильно убывает по закону Гаусса. Однако $V_{k}=a^{2} / l_{0}$ характеризует среднюю скорость движения температуры (температурных волн) для ЛУТ. Обычно она весьма мала. Очевидно, для радиации $V_{\sigma}(x, t)=-(\partial u / \partial t) /(\partial u / \partial x)$ или $V_{\sigma}(x, t)=4 \sigma \varepsilon T^{3}(x, t) / w(x)$. Область применимости уравнения (1) теперь можно определить как $V_{\sigma}(x, t)<c$, что ограничивает диапазон температур в модели: $T<\sqrt[3]{c a^{2} /(4 \sigma)}$. Если же к плотности энергии вещества добавить лучистую плотность энергии $E_{p}=4 \sigma T^{4} / c$, то сразу имеем ограничение $V_{\sigma}(x, t)<c$. Аналогичное ограничение имеет место и для УЛТ, где скорость определена как $V_{c}=16 a^{2} l l_{0} \sigma u^{3}\left(1 / 3+l_{0} / l_{1}\right)$. Кроме того, для выполнения условия равновесности длина $l_{0}$ и скорость движения температуры не могут быть слишком большими. При $l_{0} \rightarrow \infty$ равновесность нарушается. Действительно, при ограниченной второй производной $0<\left|u^{\prime \prime}\right|<\infty$ это означает бесконечно крутое изменение температуры, то есть бесконечно большую плотность потока $-k u^{\prime}$ и скорость $V_{\sigma}(x, t)$. В этом случае имеется скачок температуры, решение разрывное, и следует ставить граничные условия для излучения Стефана-Больцмана. Если же $0<\left|u^{\prime}\right|<\infty$, а $u^{\prime \prime} \rightarrow 0$, то $u^{\prime}=$ const, и имеем линейное распределение температуры. Оно не может быть стационарным в области $0<x<L$ ни при задании температур (термостатов) на границах, ни при задании тепловых потоков. Выполняя интегрирование величины $k u^{\prime \prime}-4 \sigma u^{4} u^{\prime}$, видим, что поток в область равен $\sigma T^{4}(0)-$ $-\sigma T^{4}(L)$, что при больших перепадах температуры при малых $L$ приводит к резкому ее нагреву. Таким образом, область применимости НУТ можно охарактеризовать как исследование медленных температурных процессов, когда излучение можно считать квазиравновесным, температуру локально установившейся, а скорость температурных волн существенно меньше скорости света. Далее именно такие процессы и будут рассмотрены. Рассматривать НУТ следует, когда тепловой поток за счет излучения сравним или больше, чем тепловой поток за счет теплопроводности. Это обычно имеет место при высоких температурах и ее больших градиентах. Внутри почти черного тела обычно $\delta \varepsilon(\mathbf{r}) \approx 0$, поэтому вклад от нелинейного члена достаточно мал и существенно проявляется в приповерхностном слое. Вводя для однородного сферического тела радиуса $R$ усредненную по распределению Планка длину пробега излучения $l(r)$, нетрудно получить $\delta \varepsilon(r) \approx 2 \exp (-R / l(r)) \sinh (r / l(r)) \approx \exp (-(R-r) / l(r))$. Формула показывает, что около центра тела величина $\delta \varepsilon(r)$ экспоненциально мала, а у поверхности $\delta \varepsilon(r) \approx 1$. Примером хорошо прозрачной области с $\delta \varepsilon(r) \approx 1$ может служить солнечная корона. 


\section{2. Численное интегрирование нестационарных уравнений}

Хотя численное интегрирование нестационарного уравнения теплопроводности и нелинейного уравнения Шредингера изложено в большом числе монографий и публикаций (см., например, [24-26]), полезно рассмотреть случай для цилиндрической и сферической задач с введенными нелинейными членами. Именно задачи такого типа наиболее интересны для теплофизики высоких температур. Для дискретизации пишем интегральное уравнение (ИУ)

$$
\begin{aligned}
u\left(r, t_{n+1}\right)= & u\left(r, t_{n}\right)+ \\
& +\int_{t_{n}}^{t_{n+1}}\left[\frac{\nabla \cdot\left(k u^{\prime}(r, t)\right)-\sigma \Phi(u(r, t) \delta \varepsilon(r, u(r, t)))}{w(r)}+\frac{F(r, t)}{w(r)}\right] d t .
\end{aligned}
$$

Здесь штрихом обозначено дифференцирование по координате. Обозначим в (4) интеграл от $F(r, t) / w(r)$ как $F_{n}(r)$, а оставшуюся (первую) часть подынтегральной функции - как $g(r, t)$. Эта функция явно зависит от $u$ и производных $u^{\prime}$ и $u^{\prime \prime}$, но на точном решении НУТ или ИУ (4) ее можно считать функцией координаты и времени. Формула (4) позволяет вычислить тепловое поле в момент $t_{n+1}$, если были известны тепловые поля $u(r, t), u^{\prime}(r, t)$ и $u^{\prime \prime}(r, t)$ в предыдущие моменты $t_{n}, t_{n-1}, \ldots, t_{0}$. Именно необходимость вычисления $u^{\prime}(r, t)$ и $u^{\prime \prime}(r, t)$ отличает одношаговые и многошаговые явные и неявные схемы, следующие из (4), от аналогичных стандартных схем численного интегрирования $[25,26]$. Начальным условием для задачи является известная функция $u\left(r, t_{0}\right)$. Зная ее, находим производные $u^{\prime}\left(r, t_{0}\right)$ и $u^{\prime \prime}\left(r, t_{0}\right)$ численным дифференцированием, используя граничные условия. Это имеет место и для последующих моментов $t_{n}$, определяемых из (4). Отметим, что $u^{\prime \prime}\left(r, t_{n}\right)$ удобнее определять из НУТ через $u\left(r, t_{n}\right)$ и $u^{\prime}\left(r, t_{n}\right)$, то есть достаточно вычислить только первую пространственную производную. Считаем, что область $0<r<R$ разбита точками $r_{m}$ так, что $r_{0}=0, r_{1}=\Delta r, \ldots, r_{m}=m \Delta r, \ldots$, $R=M \Delta r$. Обозначим $u_{m n}=u\left(r_{m}, t_{n}\right)$. Начальные значения теплового поля $u_{m 0}$ известны. Ищем решение при граничных условиях $u(0, t)=u_{0}(t)$ и $u^{\prime}(0, t)=0$ (отсутствие градиента в центре). Это позволяет определить температуру от центра к поверхности. При обратном проходе ставим на границе условия $u(R, t)=\varphi(t), u^{\prime}(R, t)=0$ (постоянство температуры в приповерхностном слое). Если же на границе задан скачок температуры, то есть $u(R, t) \neq \varphi(t)$, то используем условие, определяющее поток $\psi(t)=-k u^{\prime}(R, t)=\sigma \varepsilon u^{4}(R, t)-\sigma \varepsilon \varphi^{4}(t)$. Например, для космического аппарата в далеком космосе постоянное значение $\varphi(t)=2.73$ соответствует тепловому потоку в космический вакуум с температурой реликтового излучения $2.73 \mathrm{~K}$. Возьмем зависимость $k=k_{0}(r) u^{\alpha}(r, t)$. Такая аппроксимация удобна для учета координатной и температурной зависимости коэффициента теплопроводности ряда сред. Например, для горячей плазмы $\alpha \approx 5 / 2$. Положив $\alpha=0$, мы исключим температурную зависимость. Для сильно нагретых тел температуру удобно измерять по энергетической шкале $u=k_{B} T$, что требует перенормировки коэффициентов в НУТ. В сферическом случае

$$
g(r, t)=\frac{r^{-2}\left(r^{2} k_{0}(r) u^{\alpha}(r, t) u^{\prime}(r, t)\right)^{\prime}-\sigma \Phi(u(r, t), \delta \varepsilon(r, u(r, t)))}{w(r)} .
$$

Как обычно, порядок точности и тип схемы интегрирования (явная или неявная) зависит от аппроксимации функции $g(r, t)$ в (4). Используя аппроксимацию (экстраполяцию) по $m$ точкам $t_{n+1-i}$, получаем $m$-шаговые схемы, явные типа Адамса-Башфорта $(i=1,2, . ., m)$, или неявные типа Адамса-Мултона $(i=0,1, . ., m-1)$. Для рассматриваемых достаточно жестких задач более предпочтительными и устойчивыми являются неявные схемы [24]. Одношаговые $p$-этапные явные схемы типа Рунге-Кутты получаются аппроксимацией на отрезке $\left(t_{n}, t_{n+1}\right)$ 
$p$ промежуточными точками. Так, использование формулы Симпсона со схемой предикторкорректор приводит к явному методу Рунге-Кутты 4-го порядка [6]. Простейшая линейная аппроксимация $g(r, t) \approx\left[-g\left(r, t_{n-1}\right)\left(t-t_{n}\right)+g\left(r, t_{n}\right)\left(t-t_{n-1}\right)\right] / \Delta t$ и формула трапеций приводят к неявной схеме второго порядка. В случае явной схемы интеграл в (4) принимает вид $\Delta t\left(3 g\left(r, t_{n}\right)-g\left(r, t_{n-1}\right)\right) / 2+F_{n}(r)$. Для нее

$$
u\left(r, t_{n+1}\right)=u\left(r, t_{n}\right)+\Delta t\left(3 g\left(r, t_{n}\right)-g\left(r, t_{n-1}\right)\right) / 2+F_{n}(r) .
$$

Это двушаговая явная схема типа Адамса-Башфорта второго порядка по $\Delta t$. Удобно использовать явные и неявные схемы четвертого порядка, поскольку после того как вычислена функция $u\left(r, t_{n}\right)$, ее пространственные производные внутри области можно вычислять также по формулам четвертого порядка точности

$$
\begin{aligned}
u_{m(n+1)}^{\prime}=(12 \Delta r)^{-1}\left(u_{(m-3)(n+1)}-27 u_{(m-1)(n+1)}+27 u_{(m+1)(n+1)}-u_{(m+3)(n+1)}\right) & \\
u_{m(n+1)}^{\prime}=(12 \Delta r)^{-1}( & -u_{(m-2)(n+1)}+ \\
& \left.+16 u_{(m-1)(n+1)}-30 u_{m(n+1)}+16 u_{(m+1)(n+1)}-u_{(m+2)(n+1)}\right) .
\end{aligned}
$$

Вычисление пространственных производных можно уточнить. Из (2) следует

$$
\nabla \cdot\left(k u^{\prime}(r, t)\right)=w u(r, t)-F(r, t)+\sigma \Phi(u(r, t), \varepsilon(r, u(r, t))) .
$$

Интегрируя это уравнение по сферическому слою $r_{m}<r<r_{m+1}$ для $t=t_{n+1}$, имеем

$$
\begin{aligned}
& u^{\prime}\left(r_{m+1}, t_{n+1}\right)-u^{\prime}\left(r_{m}, t_{n+1}\right) \frac{r_{m}^{2} k\left(r_{m}\right)}{r_{m+1}^{2} k\left(r_{m+1}\right)}= \\
& \quad=\frac{1}{r_{m+1}^{2} k\left(r_{m+1}\right)} \int_{r_{m}}^{r_{m+1}}\left[w(r) u\left(r, t_{n+1}\right)-F\left(r, t_{n+1}\right)+\sigma \Phi(u(r, t), \delta \varepsilon(r, u(r, t)))\right] r^{2} d r .
\end{aligned}
$$

Подставляя $w(r) \dot{u}\left(r, t_{n+1}\right)=r^{-2}(\partial / \partial r)\left(r^{2} k u^{\prime}\left(r, t_{n+1}\right)\right)+F\left(r, t_{n+1}\right)+\sigma \Phi(u(r, t), \delta \varepsilon(r, u(r, t))) r^{2} d r$, имеем тождество, то есть формула точна для $u^{\prime}\left(r, t_{n+1}\right)$. Интегрируя (7) еще раз, получаем

$$
u\left(r_{m+1}, t_{n+1}\right)=u\left(r_{m}, t_{n+1}\right)+\left[r_{m+1}^{2} k\left(r_{m+1}\right)\right]^{-1} \int_{r_{m}}^{r_{m+1}} r^{2} k(r) u^{\prime}\left(r, t_{n+1}\right) d r .
$$

Используя в (8) формулу трапеций, имеем

$$
\begin{array}{r}
u_{(m+1)(n+1)}=u_{m(n+1)}+\Delta r\left[r_{m+1}^{2} k\left(r_{m+1}\right)\right]^{-1}\left(r_{m+1}^{2} k\left(r_{m+1}\right) u_{(m+1)(n+1)}^{\prime}+\right. \\
\left.+r_{m}^{2} k\left(r_{m}\right) u_{m(n+1)}^{\prime}\right) / 2 .
\end{array}
$$

Повысить точность можно используя квадратичную или кубичную аппроксимацию подынтегральной функции в (8). Так, интегрируя (8) по частям, имеем

$$
u\left(r_{m}, t_{n+1}\right)=\left[r_{m+1}^{2} k\left(r_{m+1}\right)\right]^{-1}\left(r_{m}^{2} k\left(r_{m}\right) u\left(r_{m}, t_{n+1}\right)+\int_{r_{m}}^{r_{m+1}}\left(r^{2} k(r)\right)^{\prime} u\left(r, t_{n+1}\right) d r\right) .
$$


Вычисляя интеграл по формуле трапеций и используя разложение $k\left(r_{m}\right) \approx k\left(r_{m+1}\right)-\Delta r k^{\prime}\left(r_{m+1}\right)$, получаем

$$
\begin{aligned}
& u_{(m+1)(n+1)}= \\
& \quad=u_{m(n+1)} \frac{2 r_{m+1} k\left(r_{m}\right)-\Delta r k\left(r_{m+1}\right)+k^{\prime}\left(r_{m+1}\right)\left(r_{m+1}^{2}+\Delta r^{2}\right)-r_{m}\left(k\left(r_{m}\right)+r_{m} k^{\prime}\left(r_{m}\right) / 2\right)}{r_{m+1} k\left(r_{m+1}\right)+r_{m+1}^{2} k^{\prime}\left(r_{m+1}\right) / 2} .
\end{aligned}
$$

Соотношения записаны для случая зависимости коэффициента теплопроводности от координаты. Для устойчивости шаги $\Delta t$ и $\Delta r$ следует согласовывать [24]. Заметим, что, если $k=0$, производная второго порядка не возникает, и в этом случае для производной $u^{\prime}$ можно использовать как формулы дифференцирования, так и определять ее из НУТ. Такое НУТ следует использовать для хорошо прозрачных и очень сильно нагретых сред с относительно малой теплопроводностью.

\section{3. Нелинейные температурные волны}

Рассмотрим применение нестационарного нелинейного интегрального уравнения к задаче о движении температурной волны для стандартной одномерной задачи в декартовых координатах о распространении тепла в слое $0<x<L$ между двумя термостатами, то есть при граничных условиях $u(0, t)=T_{1}, u(L, t)=T_{2}$ и начальном условии $u\left(x, t_{0}\right)=u_{0}(x)$. Считаем слой достаточно тонким, хорошо прозрачным и расположенным между двумя полупространствами. Величину $\delta \varepsilon(x)$ считаем постоянной (что возможно при не слишком большой толщине, слабо влияющей на коэффициент черноты), $T_{1}<T_{2}$. Начальный тепловой поток $k(0) u_{0}^{\prime}(0)-k(L) u_{0}^{\prime}(L)$ за счет теплопроводности из термостатов в рассматриваемую область считаем равным нулю, то есть постулируем отсутствие градиентов температуры у поверхностей слоя: $u_{0}^{\prime}(0)=u_{0}^{\prime}(L)=0$. Далее для простоты считаем, что коэффициент теплопроводности слоя $k$ постоянен и не имеет разрывов: $k(0)=k, k(L)=k$. Имеется независимый от времени радиационный тепловой поток $\sigma\left(\delta \varepsilon(0) T_{1}^{4}-\delta \varepsilon T_{2}^{4}\right)$. Вводим безразмерную координату $\chi=x / L$ и безразмерное время $\tau=t / t_{0}$. Здесь $t_{0}=L^{2} / a^{2}$ - характерное время движения температуры в области. Мы пренебрегаем плотностью лучистой энергии, поэтому $a^{2}=k /\left(c_{0} \rho\right)=$ const. Обозначаем $\tilde{\sigma}=\delta \varepsilon \sigma, a^{2}=a^{2} L^{2} / t_{0}$ и вводим характеристическую температуру $u_{c}=\sqrt[3]{c \rho L /\left(4 \tilde{\sigma} t_{0}\right)}=\sqrt[3]{k /(4 \tilde{\sigma} L)}$, нормированную безразмерную температуру $U(\chi, \tau)=u(\chi, \tau) / u_{c}$ и нормированную безразмерную плотность мощности источников $\tilde{f}_{0}(\chi, \tau)=t_{0} f_{0}\left(\chi L, \tau t_{0}\right) / u_{c}$. Тогда имеем безразмерное НУТ вида

$$
\partial_{\tau} U(\chi, \tau)=\tilde{a}^{2} \partial_{\chi}^{2} U(\chi, \tau)-U^{3}(\chi, \tau) \partial_{\chi} U(\chi, \tau)+\tilde{f}_{0}(\chi, \tau)
$$

Величина $V(U)=U^{3}(\chi, \tau)$ характеризует безразмерную скорость, пропорциональную кубу температуры. Далее рассмотрен случай $\tilde{f}_{0} \equiv 0$. Можно непосредственно интегрировать (11), но мы поступим другим образом. Ищем решение в виде $U(\chi, \tau)=U(\chi, 0)+\tilde{U}(\chi, \tau)$, что позволяет написать НУТ вида

$$
\partial_{\tau} \tilde{U}(\chi, \tau)=\tilde{a}^{2} \partial_{\chi}^{2} \tilde{U}(\chi, \tau)+\tilde{f}_{0}(\chi, \tau)+\Psi(\chi, \tau) .
$$

Здесь мы отнесли нелинейный член к источникам

$$
\Psi(\tilde{U}(\chi, \tau))=-[\tilde{U}(\chi, \tau)+U(\chi, 0)]^{3} \partial_{\chi}(\tilde{U}(\chi, \tau)+U(\chi, 0))
$$

Решение НУТ (12) удовлетворяет условиям $\tilde{U}(0, \tau)=\tilde{U}(1, \tau)=0$ и $\tilde{U}(\chi, 0)=0$ в силу его построения. 
Для уравнения (12) с нулевыми граничными и начальным условиями при заданной плотности источников имеет место решение, определяемое формулой Грина [1]

$$
\begin{aligned}
& \tilde{U}(\chi, \tau)=\int_{0}^{\tau} \int_{0}^{1} G\left(\chi, \chi^{\prime}, \tau-t a u^{\prime}\right)\left[\tilde{f}_{0}\left(\chi^{\prime}, \tau^{\prime}\right)+\Psi\left(\tilde{U}\left(\chi^{\prime}, \tau^{\prime}\right)\right)\right] d \chi^{\prime} d \tau^{\prime}, \\
& G\left(\chi, \chi^{\prime}, \tau\right)=2 \theta(\tau) \sum_{n=1}^{\infty} \exp \left(-(n \pi \tilde{a})^{2} \tau\right) \sin (n \pi \chi) \sin \left(n \pi \chi^{\prime}\right) .
\end{aligned}
$$

Здесь $\theta(\tau)-$ функция Хэвисайда. ФГ (15) удовлетворяет уравнению $\left(\partial_{\tau}-\tilde{a}^{2} \partial_{\chi}^{2}\right) G\left(\chi, \chi^{\prime}, \tau\right)=$ $=\delta(\tau) \delta\left(\chi-\chi^{\prime}\right)$ и нулевым граничным условиям. Подействуем оператором $\partial_{\tau}-\tilde{a}^{2} \partial_{\chi}^{2}$ на (14). Дифференцируя (13) по верхнему пределу $\tau$, получаем ноль в силу (15) и свойства $\theta(0)=0$. Действуя оператором под интегралом с учетом $\partial_{\tau} \theta(\tau)=\delta(\tau)$ и равенства при $\tau=0$ удвоенной суммы в (15) дельта-функции $\delta\left(\chi-\chi^{\prime}\right)$, получаем исходное уравнение (12). Подставляя (13) в (14), имеем нелинейное интегродифференциальное уравнение

$$
\begin{aligned}
& \tilde{U}(\chi, \tau)=\bar{U}_{0}(\chi, \tau)+\int_{0}^{\tau} \int_{0}^{1} G\left(\chi, \chi^{\prime}, \tau-t a u^{\prime}\right) \Psi\left(\tilde{U}\left(\chi^{\prime}, \tau^{\prime}\right)\right) d \chi^{\prime} d \tau^{\prime}, \\
& \Psi\left(\tilde{U}\left(\chi^{\prime}, \tau^{\prime}\right)\right)=-(\tilde{U}(\chi, \tau)+U(\chi, 0))^{3} \partial_{\chi}(\tilde{U}(U(\chi, 0)+\chi, \tau)), \\
& \bar{U}_{0}(\chi, \tau)=\int_{0}^{\tau} \int_{0}^{1} G\left(\chi, \chi^{\prime}, t^{\prime}\right) \tilde{f}_{0}\left(\chi^{\prime}, t^{\prime}\right) d \chi^{\prime} d t^{\prime} .
\end{aligned}
$$

При наличии источников (18) дает вклад в нулевое приближение. В нашем случае источников нет, $\bar{U}_{0} \equiv 0$. Для решения ИДУ (16) вводим дискретные времена и представляем первый интеграл в (29) по квадратурным формулам с весовыми коэффициентами $c_{m}$

$$
\tilde{U}_{n}(\chi)=\tilde{U}\left(\chi, \tau_{n}\right)=\sum_{m=0}^{n} c_{m} \int_{0}^{1} G\left(\chi, \chi^{\prime}, \tau_{n}-\tau_{m}\right) \Psi\left(\tilde{U}_{m}\left(\chi^{\prime}\right)\right) d \chi^{\prime}
$$

Поскольку $\tilde{U}_{0}(\chi)=0$, формула (19) позволяет последовательно вычислить все функции $\tilde{U}_{n}(\chi)$. Они входят в нее неявно и нелинейно, поэтому вычисление представляет собой итерационный процесс. Для его реализации надо вычислять функцию (17) и интеграл. Его также вычисляем методом квадратур, вводя дискретные координаты $\chi_{s}=s \Delta \chi$, где $s=0,1, \ldots, S$. Для первой итерации в правой части $\tilde{U}_{n}(\chi)=\tilde{U}_{n-1}(\chi)$. Таким образом, (19) приобретает вид

$$
\tilde{U}_{n r}=\tilde{U}\left(\chi_{r}, \tau_{n}\right)=\sum_{m=0}^{n} c_{m} \sum_{s=0}^{S} c_{s} G\left(\chi_{r}, \chi_{s}, \tau_{n}-\tau_{m}\right) \Psi\left(\tilde{U}_{m s}\right) .
$$

Итерации в (20) необходимо проводить до сходимости. При вычислении производной в $\Psi$ во внутренней области использованы формулы четвертого порядка точности. Для интегрирования в (20) были использованы формулы трапеций (второй порядок точности) и Симпсона (четвертый порядок точности). Второй случай также реализуется из первого применением метода РунгеРомберга на стандартной сетке и на сетке с двойным шагом. Массивы в (20) запоминались и затем итерационно уточнялись. Матрицу $G$ достаточно вычислить один раз. 


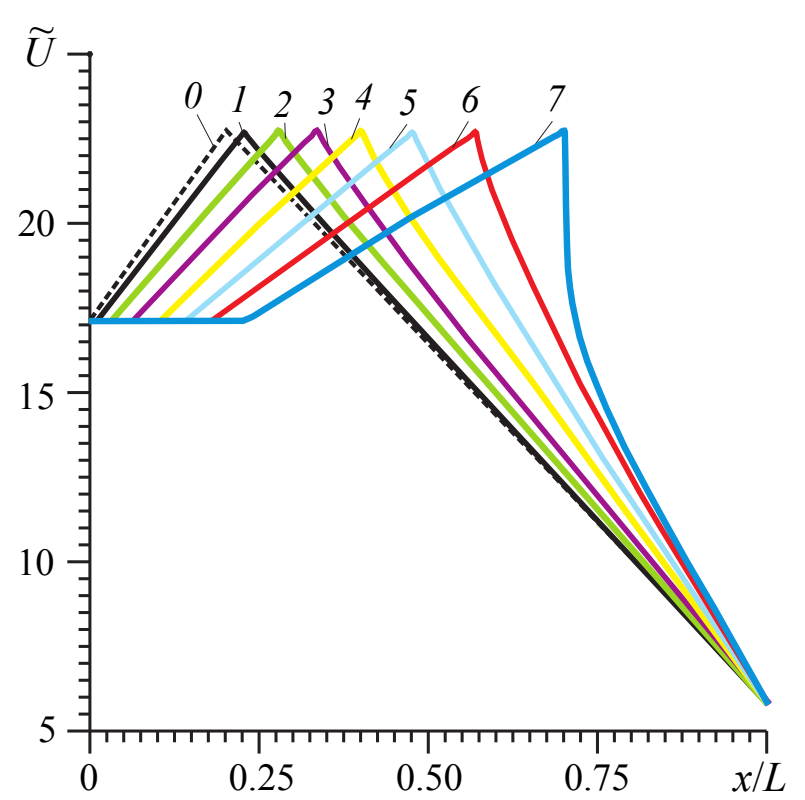

Рис. 1. Движение треугольного импульса температуры между двумя термостатами: кривые $0,1,2,3,4,5,6,7$ соответствуют моментам времени $0,2.18 \cdot 10^{-6}, 4.36 \cdot 10^{-6}$, $8.72 \cdot 10^{-6}, 1.32 \cdot 10^{-5}, 2.18 \cdot 10^{-5}, 1.6 \cdot 10^{-5}, 3.2 \cdot 10^{-5}$ с

Fig. 1. Movement of a triangular pulse of temperature between two thermostats: curves $0,1,2,3,4,5,6,7$ correspond to time points $0,2.18 \cdot 10^{-6}, 4.36 \cdot 10^{-6}, 8.72 \cdot 10^{-6}, 1.32 \cdot 10^{-5}$, $2.18 \cdot 10^{-5}, 1.6 \cdot 10^{-5}, 3.2 \cdot 10^{-5} \mathrm{~s}$

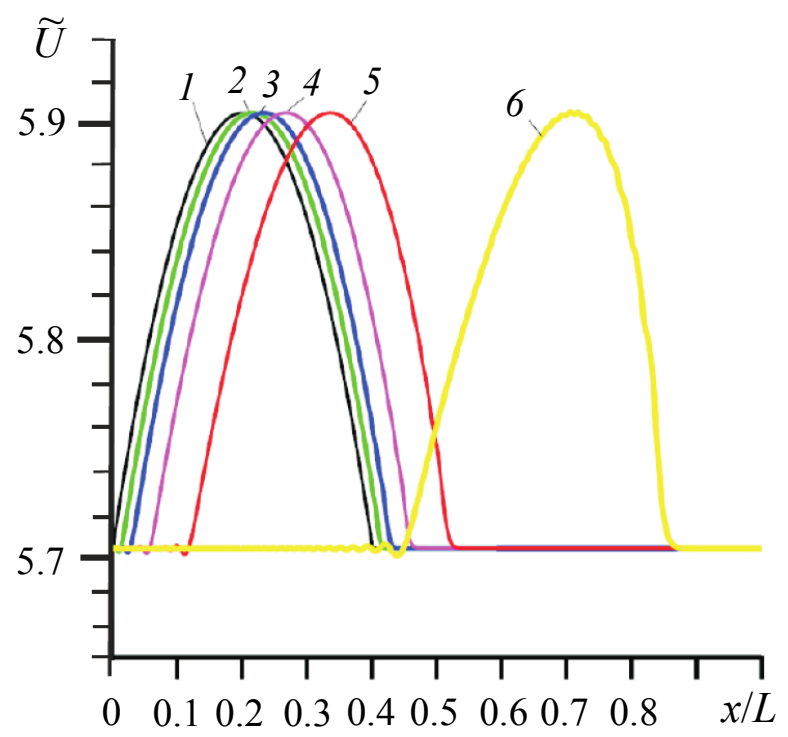

Рис. 2. Движение слабо несимметричного импульса в постоянном поле температуры: кривые $1,2,3,4,5,6$ соответствуют моментам времени $6.66 \cdot 10^{-6}, 1.33 \cdot 10^{-5}$, $6.66 \cdot 10^{-5}, 1.33 \cdot 10^{-4}, 2.66 \cdot 10^{-4}, 5.33 \cdot 10^{-4} \mathrm{c}$

Fig. 2. Motion of a weakly asymmetric pulse in a constant temperature field: curves $1,2,3,4,5,6$ correspond to moments of time $6.66 \cdot 10^{-6}, 1.33 \cdot 10^{-5}, 6.66 \cdot 10^{-5}, 1.33 \cdot 10^{-4}$, $2.66 \cdot 10^{-4}, 5.33 \cdot 10^{-4} \mathrm{~s}$
Результаты решения (16) представлены на рис. 1 в виде движения нелинейной волны для начального импульса температуры треугольной формы (кривая 0) между двумя термостатами. Такой импульс может, например, возникнуть при взрыве (быстром горении), когда плотность источников распределена по соответствующему закону. Рассмотрены следующие условия: $T_{1}=3000, T_{2}=1000$, максимальная температура $T_{\max }=4000 \mathrm{~K}$ при $x_{0}=$ $=0.2 \mathrm{м}$, при длине $L=1 \mathrm{м}, c_{0}=1000$, $\rho=1000, k=1 \mathrm{BT} /($ м $\cdot К), \delta \varepsilon=0.9$. Это соответствует $u_{c} \approx 0.2 \mathrm{~K}$. Без учета излучения $(\sigma=0)$ распределение температуры остается неподвижным, поскольку кусочно-линейное ее распределение удовлетворяет ЛУТ. В частности, возможно стационарное решение ЛУТ с начальным линейным распределением температуры $u(x, 0)=T_{1}+\left(T_{2}-T_{1}\right) \chi$. Учет излучения для него приводит к движению вправо с укручением фронта, как и для рис. 2. С течением времени волна проходит и устанавливается температура $u(x, t)=T_{1}$. При данных условиях скорость за счет излучения на порядки больше, чем за счет теплопроводности. Горб температуры движется в сторону меньшей температуры с обострением переднего фронта (рис. 1, 2). При прохождении волны устанавливается стационарное постоянное температурное поле. Как нетрудно видеть, такое решение удовлетворяет однородному уравнению (24), при этом на правой границе имеет место скачок температур и тепловой поток. Приведенный пример показывает, что возникающее между термостатами распределение температуры приводит к ее выравниванию так, что градиент отсутствует. Следует отметить, что укручение фронта с опрокидыванием типа [27] для температурной волны невозможно: температура однозначная и плавно меняющаяся со временем и в пространстве функция. В расчете не учтена плотность лучистой энергии и зависимость $\delta \varepsilon$ от координаты, что ограничивает крутизну и скорость. Для решения вопроса о применимости НУТ следует рассматривать процессы в конкретных материальных средах и вычислять усредненный по спектру пробег излучения $l$ и 
величину $\delta \varepsilon(x)$, для чего необходимо решать задачу о поглощении излучения конкретным веществом. Для плотных и хорошо нагретых тел пробег весьма мал и может достигать нанометров. Именно на такой длине $l$ температура должна изменяться слабо. В случае нарушения применимости НУТ следует использовать другие модели, например, теорию лучистого переноса.

Другой возможный подход к решению (19) - использование разложения

$$
\tilde{U}(\chi, \tau)=\sum_{m=1}^{M} a_{m}(\tau) \sin (m \pi \chi)
$$

с начальными условиями $a_{m}(0)=0$. Подставляя его в дифференциальное уравнение (12) и применяя метод Галеркина, имеем

$$
\begin{gathered}
a_{m}(\tau)=-a^{2} \alpha_{m}^{2} a_{m}(\tau)+B_{m}(\tau)+C_{m}\left(a_{1}(\tau), a_{2}(\tau), \ldots, a_{M}(\tau)\right), \\
m=1,2, \ldots, M .
\end{gathered}
$$

Это задача Коши для системы дифференциальных уравнений, в которой в общем случае

$$
\begin{gathered}
B_{m}(\tau)=2 \int_{0}^{1} \tilde{f}_{0}(\chi, \tau) \sin \left(m \pi \chi^{\prime}\right) d \chi \\
C_{m}\left(a_{1}(\tau), a_{2}(\tau), \ldots, a_{M}(\tau)\right)= \\
=2 \int_{0}^{1} \sin \left(m \pi \chi^{\prime}\right)\left\{-\left[\tilde{U}(\chi, \tau)+\tilde{U}_{0}(\chi)\right]^{3}\left[\tilde{U}^{\prime}(\chi, \tau)+\tilde{U}^{\prime}{ }_{0}(\chi)\right]+\tilde{a}^{2} \tilde{U}^{\prime \prime}{ }_{0}(\chi)\right\} d \chi .
\end{gathered}
$$

Решаем задачу Коши для точек $\tau_{n}=n \Delta \tau$. При $\tau=0$ имеем

$$
C_{m}\left(a_{1}(0),\left(a_{2}(0), \ldots, a_{M}(0)\right)=2(m \pi)^{2} \int_{0}^{1} \sin (m \pi \chi)\left[a^{2} u_{0}^{\prime \prime}(\chi)-4 \tilde{\sigma} u_{0}^{3}(\chi) u_{0}^{\prime}(\chi)\right] .\right.
$$

После каждого прохода в (21) пересчитываем $\tilde{u}(\chi, \tau)$ и $C_{m}$, а затем снова применяем такой проход. При шаге по времени целесообразно делать несколько подобных итераций. Все интегралы вычисляются аналитически, но в них возникают степени рядов в виде тройных сумм, поэтому удобнее численное интегрирование. Такой подход более сложен, чем на основе итераций (20).

Более интересен случай, когда на правом конце температура задана (термостат $T_{2}$ ), а на левом имеет место граничное условие $T_{1}(t)=\eta(t)$. В этом случае поток переменный, и распространение импульсов определяет функция $\eta(t)$. Если $\eta(t)=T_{1}(0)+\Delta T \sin (\omega t)$, то при $T_{1}(0)>T_{2}$ имеет место средний поток и его периодическое изменение. При $T_{1}(0)=T_{2}$ среднего потока нет, и режим изменения температуры - колебательный с пространственным запаздыванием. Точно так же можно задавать не температуру, а изменение потока тепла. На рис. 2 представлено движение немного несимметричного импульса температуры на уровне постоянной температуры 1000 градусов для $k=1000$ Вт/(м·К). Решение получено с использованием приведенной ниже ФГ методом итераций. При использовании ЛУТ импульс неподвижен и расплывается в обе стороны со временем. Учет излучения приводит к его ускоренному движению в сторону более крутого фронта. За счет обострения фронтов скорость движения со временем увеличивается.

Рассмотрим ФГ для распространение импульсов в бесконечной структуре $-\infty<x<\infty$, находящейся при постоянной температуре $T_{0}$. Пусть задано начальное распределение температуры импульса $u\left(x, t_{0}\right)=T_{0}+\tilde{u}_{0}(x)$, где функция $\tilde{u}_{0}(x)$ отлична от нуля в области $|x|<x_{0}$. 
Теперь координатное интегрирование идет по бесконечной области, а ФГ имеет вид

$$
G\left(x-x^{\prime}, t\right)=\frac{\theta(t)}{\left(4 \pi a^{2}|t|\right)^{1 / 2}} / \exp \left(-\left(x-x^{\prime}\right)^{2} /\left(a f^{2} t\right)\right),
$$

где $\theta(t)$ - функция Хэвисайда. Ее также можно записать в виде

$$
G(x, t)=\frac{1}{(2 \pi)^{2}} \int_{-\infty}^{\infty} \int_{-\infty}^{\infty} \frac{\exp (i \omega t-i \alpha x)}{i \omega+a^{2} \alpha^{2}} d \omega d \alpha=\frac{1}{4 \pi} \int \lim _{-\infty}^{\infty} \frac{\exp (i \omega t-(i-1) \sqrt{\omega / 2}|x| / a)}{(1+i) \sqrt{\omega / 2 a}} d \omega .
$$

Имеем спектр начального импульса

$$
U(\alpha)=\tilde{U}(\omega)=\int_{-x_{0}}^{x_{0}} \tilde{u}_{0}(x) \exp (i \alpha x) d x
$$

Поскольку $\alpha=a \sqrt{\omega / 2}[1]$, можно записать монохроматическую волну в виде

$$
U(\omega, t)=\tilde{U}(\omega) \exp (-\alpha|x| \cos (\omega t-a \sqrt{\omega} x / 2)) .
$$

Среднюю скорость расплывания импульса можно теперь определить как

$$
\bar{V}=a \sqrt{2 \omega}=\frac{a}{\sqrt{2}} \int_{-\infty}^{\infty} \omega^{1 / 2}|\tilde{U}(\omega)|^{2} d \omega .
$$

Для ограниченности (23) функция $\tilde{u}_{0}(x)$ должна быть достаточно гладкой и не иметь скачков. Так, для прямоугольного импульса $\tilde{U}(\omega) \sim \omega^{-1 / 2}$, и интеграл (24) расходится. Для треугольного импульса $\tilde{U}(\omega) \sim \omega^{-1}$, и такая скорость уже конечная. Выполняя обратное преобразование Фурье для (23), получаем нестационарную волну (импульс), которую также можно представить через ФГ [1]

$$
\tilde{u}(x, t)=\int_{0}^{t} \int_{-x_{0}}^{x_{0}} G\left(x, x^{\prime}, t\right) \tilde{u}_{0}(x) d x d \tau .
$$

Скорость температурной волны в точке $x$ в момент $t$ теперь можно определить как

$$
V(x, t)=-\frac{\partial \tilde{u}(x, t) / \partial t}{\partial \tilde{u}(x, t) / \partial x}=-\frac{\int_{-x_{0}}^{x_{0}} G\left(x, x^{\prime}, t\right) \tilde{u}_{0}(x) d x}{\int_{0}^{t} \int_{-x_{0}}^{x_{0}} G\left(x, x^{\prime}, t\right) \tilde{u}_{0}(x) d x d \tau} .
$$

Бесконечную область интегрирования заменим на конечную, пользуясь сильной локализацией ФГ (22) на малых временах.

Полученное НУТ можно использовать для моделирования температурных волн в Солнце и Земле. Для таких тел характерно весьма медленное изменение температуры со временем и чрезвычайно малая величина $\delta \varepsilon$, почти не изменяющаяся в глубине. Также температура почти не меняется на временах порядка миллиарда лет, то есть уравнения можно считать стационарными. Поэтому интерес представляют флюктуации температуры. В фотосфере Солнца можно оценить ее скорость как $V_{\sigma}(x)=2280 \mathrm{~m} / \mathrm{c}$, а в его центре за счет большой плотности она порядка $1.6 \mathrm{~mm} / \mathrm{c}$. Флуктуация температуры выйдет из центра на поверхность более чем за $3 \cdot 10^{10}$ с или 997 лет. 
Теория лучистого переноса дает еще на три порядка большее время выхода фотона из центра на поверхность за счет многократных актов его поглощения/излучения. Для земной коры при экспоненциальном распределении температуры имеем $V_{k}(x)=a^{2} \gamma \sim 10^{-16}$ м/с. Говорить о такой скорости возможно, если температура в центре изменяется весьма медленно. Для центра Земли это 100 градусов на миллиард лет. Отметим также, что в центре небесных тел $\delta \varepsilon \approx 0$, и только вблизи поверхности эта величина может существенно отличаться от нуля. Обычно температурные волны для ЛУТ рассматривают для периодического с частотой $\omega$ возбуждения на границах, и тогда фазовая скорость определяются как $V_{\omega}=a \sqrt{2 \omega}$. Такие волны затухают и обладают дисперсией. Поэтому скорость распространения (расплывания) теплового импульса или температурного волнового пакета как целого определяется неоднозначно и достаточно сложно. Один из возможных методов - усреднение фазовых скоростей по спектру с функцией спектральной плотности мощности [20].

Рассмотрим еще одну возможность применения НУТ. Она связана с моделированием квазистационарного остывания цилиндрических или сферических тел, например, Земли. Как известно, использование ЛУТ в модели остывания Земли без учета источников тепла (в основном, распад $\mathrm{K}^{40}$ в коре и гидродинамический нагрев расплавленных пород за счет вращения Земли) дает нереально быструю скорость остывания $[1,28]$. Использование же ЛУТ с таким учетом приводит к завышению более чем на порядок температуры в центре Земли (по современным косвенным оценкам это 5000-6000 К). Использование ИУ на основе НУТ позволяет получить оценку порядка $6000 \mathrm{~K}$.

Рассмотрим численный пример, где применение НУТ дает более реальный результат, чем ЛУТ. Пусть цилиндрический электрический водонагреватель с внешним радиусом $R=0.3$ м, нагретый до температуры $80^{\circ} \mathrm{C}$, отключен и начал остывать в комнате при температуре $25^{\circ} \mathrm{C}$. Цилиндр окружает теплоизоляционный слой пенополистирола толщины $\Delta r=1$ см с $k=0.05$, $\rho=150$ кг $/ \mathrm{m}^{3}$ и $c_{0}=1340$ Дж/(кг·К). Вода находится внутри цилиндра радиуса 0.29 м. Толщину металлической оболочки считаем малой и не учитываем, высоту цилиндра считаем большой и теплообмен через торцы не учитываем. Не учитываем также изменение плотности воды и теплоемкости с температурой. Принципиально задача не отличается от процесса остывания Земли, но легко решается как приближенным инженерным расчетом, так и интегрированием ЛУТ и НУТ, поскольку не требует астрономического числа шагов, а упрощение имеет место в силу почти постоянства температуры воды вдоль радиуса. Сначала рассмотрим только теплопроводность. В силу большого коэффициента теплопроводности $k_{w}$ воды и малой скорости остывания считаем распределение температуры в воде постоянным по радиусу: $T(0, t)=T(R-\Delta r)$. Определяя плотность потока тепла как $k_{w}(T(0, t)-T(R)) / \Delta r$, имеем $2 k_{w}(T(R)-T(0, t)) / \Delta r=c_{w} \rho_{w} R \dot{T}(0, t)$, или точное решение

$$
T(0, t)=T(R)+[T(0,0)-T(R)] \exp \left(-t / \tau_{k}\right),
$$

где $\tau_{k}=\Delta r R c_{w} \rho_{w} /\left(2 k_{w}\right)=1.2 \cdot 10^{5}$ с. Здесь индексом $w$ обозначены параметры воды. За сутки эта зависимость дает спад температуры до $51.6^{\circ} \mathrm{C}$ (рис. 3). Если учитывать только излучение, баланс приобретает вид ДУ $2 \sigma \varepsilon\left(t^{4}(R)-T^{4}(0, t)\right)=c_{w} \rho_{w} R \dot{T}(0, t)$, которое эквивалентно следующему нелинейному интегральному уравнению:

$$
T(0, t)=T(0,0)+T^{4}(R) t / \tau_{\sigma}-\tau_{\sigma}^{-1} \int_{0}^{t} T^{4}(0, \tau) d \tau,
$$

где $\tau_{\sigma}=c_{w} R /(2 \sigma \varepsilon)$. Даже при $\varepsilon=1$ получаем $\tau_{\sigma}=10^{13}$ с. При очень малых временах имеем нулевое приближение $T(0, t) \approx T(0,0)-\left[T^{4}(0,0)-T^{4}(R)\right] t / \tau_{\sigma}$. Если $\Delta=\left[T^{4}(0,0)-\right.$ $\left.-T^{4}(R)\right] t / \tau_{\sigma} \ll 1$, то, подставляя это значение под интеграл, получаем первое приближение 
$T(0, t)=T(0,0)-\Delta\left(1-2 T^{3}(0,0) t / \tau_{\sigma}\right)$. Оно показывает, что за 10 минут температура упадет на 0.47 градуса. Линейное приближение дает спад 0.3 градуса, то есть здесь НУТ необходимо, и учет теплопроводности и излучения дает более быстрый спад (см. рис. 3) и его вклад на начальном этапе остывания больше. Неявная одношаговая схема решения ДУ для баланса с учетом обоих типов теплопроводности имеет вид

$$
\begin{aligned}
u_{n+1}=u_{n} & +\frac{\Delta t}{\tau_{k}}\left[T(R)-u_{n}\left(1+\delta_{n} / 2\right)\right]+ \\
& +\frac{\Delta t}{\tau_{\sigma}}\left[T^{4}(R)-u_{n}^{4}\left(1+2 \delta_{n}\left(1+\delta_{n}\left(1+\delta_{n}\left(1 / 2+\delta_{n} / 10\right)\right)\right)\right)\right] .
\end{aligned}
$$

В ней $u_{n}=u\left(t_{n}\right), \Delta u_{n}=u_{n+1}-u_{n}, \delta_{n}=\Delta t \Delta u_{n} / u_{n}$. Если шаг мал и $\delta_{n} \ll 1$, то имеем условие, когда вклад обоих типов теплопроводности одинаков: $\tau_{\sigma} / \tau_{k}=\left(T(R)+u_{0}\right)\left(T^{2}(R)+u_{0}^{2}\right)$. Здесь $u_{0}=T(0,0)$. Если отношение $T(R) / u_{0}$ близко к единице, то $\tau_{\sigma} / \tau_{k} \sim 3 u_{0}^{3}$, что как раз реализуется в нашем случае. Данная задача легко интегрируется при условиях $u(R)=298$ К и определении $u^{\prime}(R, t)$ из решения ДУ для баланса. Считаем, что $u^{\prime}(R, t)=u^{\prime}(R-\Delta r, t)$. Соответствующее координатное ДУ имеет вид (положено $\delta \varepsilon=1) c_{0} \rho r \partial u(r, t) / \partial t=\left[r\left(k u^{\prime}(r, t)-\sigma u^{4}(r, t)\right)\right]^{\prime}$. Для каждого момента времени его решение следует искать обратным проходом от $R^{\prime} R-\Delta r$. Определяя функцию

$$
\varphi(r, t)=\int_{r}^{R^{\prime}} c_{0}\left(r^{\prime}\right) \rho\left(r^{\prime}\right) \dot{u}\left(r^{\prime}, t\right) r^{\prime} d r^{\prime}+\sigma R u^{4}(R)
$$

и интегрируя в пределах $\left(r, R^{\prime}\right)$, имеем ДУ в виде $u^{\prime}(r, t)=\left(R^{\prime} / r\right) u^{\prime}\left(R^{\prime}, t\right)+\left[\sigma\left(r u^{4}(r, t)-\right.\right.$ $\left.\left.-R^{\prime} u^{4}\left(R^{\prime}, t\right)\right)-\varphi(r, t)\right] /(k(r) r)$. Функция (26)

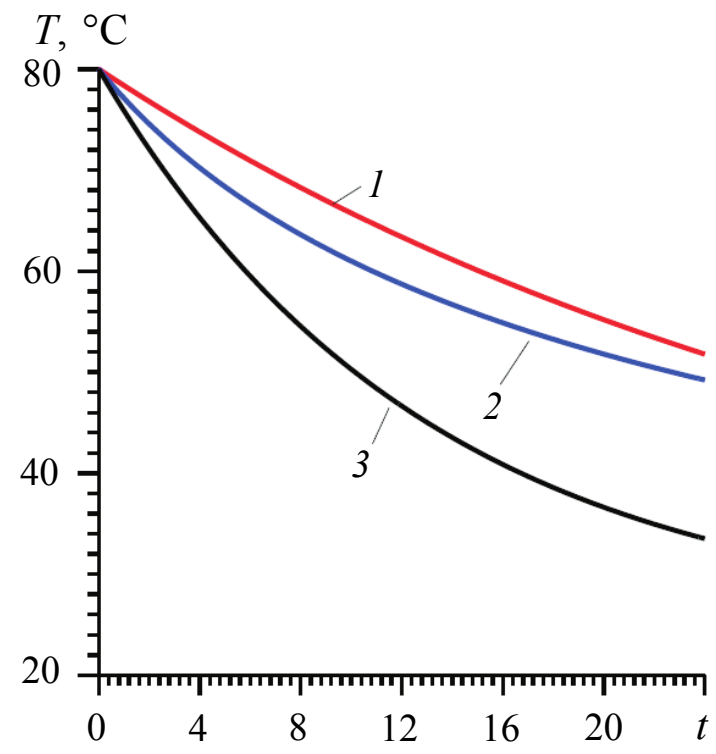

Рис. 3. Остывание нагревателя (время в часах) с учетом только теплопроводности (кривая 1), с учетом только лучистой теплопроводности (2), на основе НУТ с учетом обоих механизмов теплопередачи (3)

Fig. 3. Cooling of the heater (time in hours) taking into account only the thermal conductivity (curve 1 ), taking into account only the radiant thermal conductivity (2), based on nonlinear equation of thermal conductivity taking into account both heat transfer mechanisms (3) дает приращение энергии в конечном слое. Поскольку исходное ДУ получено без учета границ, для баланса энергии в (26) учтен обратный поток из окружающего пространства. Здесь мы считаем, что градиент на границе $R$ исчезает, а слоем $\Delta r$ в балансе можно пренебречь. Для разрешимости ДУ надо потребовать выполнение условия $u^{\prime}(0, t)=0$, то есть отсутствие градиента в центре, что физически оправдано. Это возможно, если $\sigma R^{\prime} u^{4}\left(R^{\prime}, t\right)+\varphi(r, t)=\alpha(t)+\beta(t) r$, где $\alpha(t)=k(0) R^{\prime} u^{\prime}\left(R^{\prime}, t\right)$, а $\beta(t)-$ ограниченная функция. Отсюда находим балансное уравнение

$$
\begin{aligned}
& \int_{0}^{R^{\prime}} c_{0}\left(r^{\prime}\right) \rho\left(r^{\prime}\right) \dot{u}\left(r^{\prime}, t\right) r^{\prime} d r^{\prime}= \\
& =\sigma\left[R u^{4}(R)-\right. \\
& \left.R^{\prime} u^{4}\left(R^{\prime}, t\right)\right]+ \\
& +k_{w} R^{\prime} u^{\prime}\left(R^{\prime}, t\right) .
\end{aligned}
$$

С точностью до $2 \pi$ это баланс мощности на единицу длины по оси. В случае почти полного отсутствия градиента в нагретой воде вторым членом справа можно пренебречь. Теперь 
ДУ для распределения температуры приобретает вид $u^{\prime}(r, t)=\left[\sigma u^{4}(r, t)-\beta(t)\right] / k_{w}$. Полагая $u^{\prime}\left(R^{\prime}, t\right)=\left[u\left(R^{\prime}, t\right)-u(R)\right] / \Delta r$, находим $\beta(t)=\sigma u^{4}\left(R^{\prime}, t\right)-k_{w}\left[u\left(R^{\prime}, t\right)-u(R)\right] / \Delta r$. Результаты интегрирование приведены в Таблице.

Таблица. Распределение температуры воды в ${ }^{\circ} \mathrm{C}$ вдоль радиуса Table. Distribution of water temperature in ${ }^{\circ} \mathrm{C}$ along the radius

\begin{tabular}{|c|c|c|c|c|}
\hline Время, часы & \multicolumn{4}{|c|}{ Расстояние от центра $r$, м } \\
\cline { 2 - 5 } & 0.0 & 0.1 & 0.2 & 0.29 \\
\hline 1 & 76.15 & 75.91 & 75.41 & 75.04 \\
\hline 2 & 72.44 & 72.11 & 71.76 & 71.41 \\
\hline 3 & 68.81 & 68.51 & 68.21 & 67.89 \\
\hline 4 & 65.56 & 65.27 & 64.96 & 64.65 \\
\hline 5 & 62.57 & 62.27 & 61.97 & 61.68 \\
\hline
\end{tabular}

Интегрировать можно и из центра с условием $u^{\prime}(0, t)$. При этом температуру $u(0, t)$ следует задавать приближенно. Результат интегрирования дает значение $\tilde{u}(r, t)$, которое может отличаться от $u(R, t)$. Алгоритм состоит в многократном подборе начальной температуры $u(0, t)$ для достижения минимума $|\tilde{u}(R, t)-u(R, t)|$. При этом важно, что при малых $r$ нагреватель нельзя рассматривать как абсолютно черное тело, то есть необходимо вводить зависящий от радиуса коэффициент черноты и его вариацию, например, $\delta \varepsilon(r)=1-\exp (-r / l)$. Это означает, что нет радиационного потока из центра. Только в этом случае условие $u^{\prime}(0, t)=0$ выполнено.

\section{Заключение}

Предложены новые нелинейные уравнения теплопроводности, полученные на основе закона сохранения энергии и учитывающие интегральный радиационный теплоперенос за счет излучения по закону Стефана-Больцмана. Уравнения естественным образом описывают теплопроводность в системе с вакуумными зазорами то есть с областями, где коэффициент теплопроводности обращается в нуль, но имеется тепловое поле. Характерный пример здесь - теплообмен тела, отделенного от внешней среды термоизолирующей оболочкой, когда не нужно ставить граничное условие в виде задания потока. Получены условия для температуры и ее градиента, когда уравнения применимы. Они связаны с ограничением температуры и ее градиента (скоростью температурной волны) и с квазиравновесностью процессов, когда применимо понятие температуры. Ограничение скорости волны имеет место при учете плотности радиационной энергии. Рассмотрены уравнения в декартовой, цилиндрической и сферической системах координат, условия, когда необходим учет введенных нелинейных членов, нелинейные нестационарные температурные волны, скорости переноса температуры. Отметим, что основная задача при использовании нелинейного уравнения теплопроводности состоит в определении зависимости вариации коэффициента черноты тела от координат и времени.

\section{Библиографический список}

1. Тихонов А.Н. Самарский А.А. Уравнения математической физики. М.: Наука, 1977. 736 с.

2. Патанкар C. Численные методы решения задач теплообмена и динамики жидкости / Перевод с английского под редакцией В.Д. Виленского. М.: Энергоатомиздат, 1984. $512 \mathrm{c}$.

3. Шашков А.Г., Бубнов В.А., Яновский С.Ю. Волновые явления теплопроводности. М: УРСС, 2004. $298 \mathrm{c}$.

4. Дульнев Г.Н., Парфенов В.Г., Сигалов А.В. Применение ЭВМ для решения задач теплообмена. М.: Высшая школа, 1990. 207 с.

5. Кутателадзе С.С. Основы теории теплообмена. М: Атомиздат, 1979. 416 с. 
6. Свешников А.Г., Боголюбов А.Н., Кравиов В.В. Лекции по математической физике: Учеб. пособие. М.: Изд-во МГУ, 1993. 352 с.

7. Оцисик М.Н. Сложный теплообмен. М: Мир, 1976. 615 с.

8. Адрианов В.Н. Основы радиационного и сложного теплообмена. М.: Энергия, 1972.

9. Мучник Г.Ф., Рубашов И.Б. Методы теории теплообмена. Ч. 2. Тепловое излучение. М.: Высшая школа, 1974. 270.

10. Зигель Р., Хауэлл Дж. Теплообмен излучением. М.: Мир, 1975. 934 с.

11. Спэрроу Э.М., Сесс Р.Д. Теплообмен излучением / Пер. с англ. Л., 1970. 295.

12. Унзольд А. Физика звездных атмосфер. М.: Государственное издательство иностранной литературы, 1949. $630 \mathrm{c}$.

13. Амбариумян В.А., Мустель Э.Р., Северный А.Б., Соболев В.В. Теоретическая астрофизика / Ред. В.А. Амбарцумян. М.: Гостехиздат, 1952. 635 с.

14. Чандрасекар C. Перенос лучистой энергии / Перевод с английского. Ред. Е.С. Кузнецов. М.: ИЛ, 1953. $433 \mathrm{c.}$

15. Иванов В.В. Перенос излучения и спектры небесных тел. М.: Наука, 1969. 472 с.

16. Фаворский О.Н., Каданер Я.С. Вопросы теплообмена в космосе. М.: Высш. школа, 1972. $280 \mathrm{c}$

17. Адзерихо К.С. Лекции по теории переноса лучистой энергии / Под ред. М.А. Ельяшевича. Минск, изд-во БГУ, 1975. $192 \mathrm{c.}$

18. Апресян Л.А., Кравцов Ю.А. Теория переноса излучения: Статистические и волновые аспекты. М.: Наука, 1983. 216 с.

19. Нагирнер Д.И. Лекции по теории переноса излучения: Учеб. пособие. СПб.: Изд-во СПб. ун-та, 2001. 284 с.

20. Зельдович Я.Б., Райзер Ю.П. Физика ударных волн и высокотемпературных гидродинамических явлений. М.: Наука, 1966, 688 с.

21. Давидович М.В., Корнев И.А., Тимофеев А.И. Нелинейная динамика теплопереноса в цилиндрических и сферических структурах // Вопросы прикладной физики. Межвузовский научный сборник. Саратов, 2015. С. 93-98.

22. Давидович М.В., Корнев И.А., Тимофеев А.И., Явчуновский В.Я. Одномерная цилиндрическая тепловая задача без начальных условий // Вопросы прикладной физики. Межвузовский научный сборник. Саратов, 2015. С. 35-37.

23. Лифшии Е.М., Питаевский Л.П. Физическая кинетика. М.: Наука, 1979. 528 с.

24. Ортега Дж., Пул У. Введение в численные методы решения дифференциальных уравнений. М.: Наука, 1986. 288 с.

25. Каханер Д., Моулер К., Нэш С. Численные методы и программное обеспечение. М.: Мир, 1998. $559 \mathrm{c}$.

26. Амосов А.А., Дубинский Ю.А., Копченова Н.В. Вычислительные методы для инженеров. М.: Высшая школа, 1994. 544 с.

27. Заславский Г.М., Сагдеев Р.3. Введение в нелинейную физику: От маятника до турбулентности и хаоса. М.: Наука, 1988. 368 с.

28. Тихонов А.Н. О влиянии радиоактивного распада на температуру земной коры. Изв. АН СССР, отд. матем. и естеств. наук, 1937. С. 431-459.

\section{References}

1. Tikhonov A.N., Samarskii A.A. Equations of Mathematical Physics. Moscow: Nauka, 1977. 736 p. (in Russian). 
2. Patankar S. Numerical Methods for Solving Problems of Heat Transfer and Fluid Dynamics. Translation from English edited by V.D. Vilensky. Moscow: Energoatomizdat, 1984. 512 p. (Patankar, Suhas V. Numerical Heat Transfer and Fluid Flow. Washington: New York: Hemisphere; McGraw-Hill, cop. 1980.)

3. Shashkov A.G., Bubnov V.A., Yanovsky S.Yu. Wave Phenomena of Thermal Conductivity. Moscow: URSS, 2004. 298 p. (in Russian).

4. Dulnev G.N., Parfenov V.G., Sigalov A.V. Application of Computers for Solving Heat Transfer Problems. Moscow: Higher school, 1990. 207 p. (in Russian).

5. Kutateladze S.S. Fundamentals of the Theory of Heat Transfer. M: Atomizdat, 1979, 416 p. (in Russian).

6. Sveshnikov A.G., Bogolyubov A.N., Kravtsov V.V. Lectures on Mathematical Physics: Studies. benefit. Moscow: Moscow State University, 1993. 352 p. (in Russian).

7. Ozisik M.N. Radiative Transfer and Interactions with Conduction and Convection. C. John Wiley, 1973.

8. Adrianov V.N. Fundamentals of Radiation and Complex Heat Transfer. Moscow: Energia, 1972. (in Russian).

9. Muchnik G.F., Rubashov I.B. Methods of Heat Transfer Theory. Part 2. Thermal Radiation. Moscow: Higher School, 1974. 270 p. (in Russian).

10. Siegel R., Howell J. Thermal Radiation Heat Transfer. McGraw-Hill Book Company, New York Google Scholar, 1972. (Siegel, Robert; Howell, John R. Thermal radiation heat transfer New Jourk a. o., 1972).

11. Sparrow E.M., Sess R.D. Heat transfer by radiation, Trans. from English, Leningrad, 1970. 295. (Sparrow E.M. and Cess R.D., 1978, Radiative Heat Transfer, McGraw-Hill.Google Scholar. Tien, C.-L., 1988).

12. Unsold A. Physics of Stellar Atmosphere. 2ed. Springer, Berlin, 1955.

13. Ambartsumyan V.A., Mustel E.R., Severniy A.B., Sobolev V.V. Theoretical Astrophysics. Ed. V.A. Ambartsumyan. Moscow: Gostekhizdat, 1952. 635 p. (in Russian).

14. Chandrasekhar S. Radiative Transfer. 2ed. Dover Publications Inc. 1960.

15. Ivanov V.V. Radiation Transfer and Spectra of Celestial Bodies, Moscow: Nauka, 1969. 472 p. (in Russian).

16. Favorskii O.N., Kadaner J.S. Issues of Heat Transfer in Space. M.: Higher School, 1972. 280 p. (in Russian).

17. Adzerikho K.S. Lectures on the Theory of Transfer of Radiant Energy, ed. M. A. Elyashevich, Minsk, BSU publishing house, 1975, 192 p. (in Russian).

18. Apresyan L.A., Kravtsov Yu.A. Theory of Radiation Transfer: Statistical and Wave Aspects, Moscow: Nauka, 1983, 216 p. (in Russian).

19. Nagirner D.I. Lectures on the Theory of Radiation Transfer: Studies benefit, SPb.: Publishing house SPb. Univ., 2001, 284 p.(in Russian).

20. Zeldowich Ya.B., Raizer Yu.P. Physics of Shock Waves and High-temperature Hydrodynamics Phenomena. Moscow: Nauka, 1966, 688 p. (in Russian).

21. Davidovich M.V., Kornev I.A., Timofeev A.I. Nonlinear dynamics of heat transfer in cylindrical and spherical structures. In: Questions of applied physics. Intercollegiate scientific collection. Saratov, 2015. pp. 93--98. (in Russian).

22. Davidovich M.V., Kornev I. A., Timofeev A.I., Yavchunovsky V.Ya. One-dimensional cylindrical thermal problem without initial conditions. In: Questions of applied physics. Intercollegiate Scientific Collection. Saratov, 2015. C. 35--37. (in Russian).

23. Lifshits E.M., Pitaevsky L.P. Physical Kinetics. Moscow: Nauka, 1979, 528 p. (in Russian). 
24. Ortega J., Poole W. An Introduction to Numerical Methods for Differential Equations. Pilman, Marshfield, 1981.

25. Kahaner D., Mouler C., Nash S. Numerical Methods and Software. Prentice-Hall, 1989.

26. Amosov A.A., Dubinsky Yu.A., Kopchenova N. In. Computational Methods for Engineers. Moscow: Higher school, 1994. 544 p. (in Russian).

27. Zaslavsky G.M., Sagdeev R.Z. An Introduction to Nonlinear Physics: From Pendulum to Turbulence and Chaos. Moscow: Nauka, 1988, 368 p. (in Russian).

28. Tikhonov A.N. On the influence of radioactive decay on the temperature of the Earth crust. Proc. of USSR Academy of Sciences, Mathematics and Nature Sciences, 1937, pp. 431-459 (in Russian).

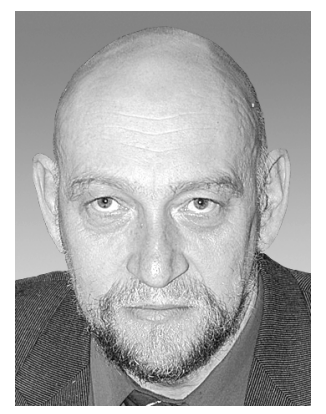

Давидович Михаил Владимирович - родился в Саратове (1950). Окончил физический факультет Саратовского государственного университета по специальности физика (специализация - теоретическая и ядерная физика, 1972). Около 20 лет проработал на предприятиях электронной промышленности г. Саратова. Защитил диссертацию на соискание ученой степени кандидата физико-математических наук в области радиофизики, включая квантовую радиофизику (1991) и доктора физико-математических наук в области применения вычислительной техники, математического моделирования и математических методов в научных исследованиях (2000). Профессор кафедры радиотехники и электродинамики СГУ, профессор (2002), член IEEE (1995), Senior Member, IEEE (2001). Область научных интересов: решение краевых задач методом интегральных и интегродифференциальных уравнений с использованием конечных элементов, вариационные методы, электродинамика и оптика структур, теория метаматериалов, решение обратных задач, микроволновое зондирование, нестационарная нелинейная электродинамика структур и диспергирующих сред, включая распространение импульсов. Автор более 250 работ по указанным направлениям, опубликованных в отечественной и зарубежной печати.

Россия, 410012 Саратов, ул. Астраханская, 83

Саратовский государстввенныйй университет им. Н.Г. Чернышеского

E-mail: Davidovichmv@info.sgu.ru

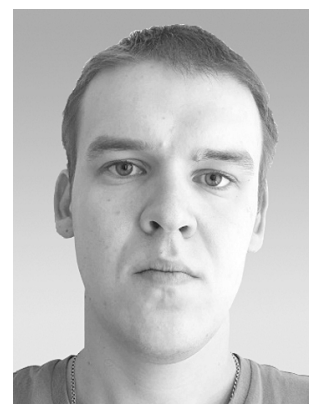

Корнев Игорь Александрович - родился в 1993 году. Окончил факультет нано-и биомедицинских технологий Саратовского государственного университета (2014) и аспирантуру СГУ по специальности радиофизика (2018). Область научных интересов - вопросы оптимизации параметров суперконденсаторных накопителей энергии и их встраивания в силовые цепи подвижных средств электрического транспорта. Автор 10 научных работ, 6 авторских свидетельств.

Россия, 410040 Саратов, Вишневая, 11

ООО НПП «Этна Плюс»

E-mail: iak.etna@Dyandex.ru

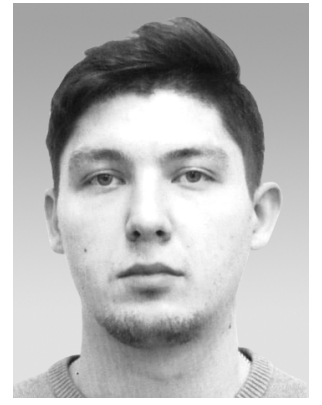

Тимофеев Антон Игоревич - родился в 1992 году. Окончил физический факультет Саратовского государственного университета (2014) и аспирантуру СГУ по специальности радиофизика (2018). Область научных интересов - электромагнитные и теплофизические процессы, протекающие в силовых цепях электрического транспорта. Автор 12 научных работ, 5 авторских свидетельств.

Россия, 410040 Саратов, Вишневая, 11

ООО НПП «Этна Плюс»

E-mail: iak.etna@Dyandex.ru 